Ann. Biol. anim. Bioch. Biophys., 1962, 2 (I), 5-1I.

\title{
ACTION DES HORMONES THYROÏDIENNES SUR LA \\ CROISSANCE EN LONGUEUR DU BRIN DE LAINE
}

\author{
Christiane THERIEZ et J. ROUGEOT \\ Station de Recherches de Physiologie animale, \\ Centre national de Recherches zootechniques, Jouy-en-Josas (Seine-et-Oise).
}

SOMMAIRE

Grâce à la radiocystine, nous avons suivi avec précision la vitesse de croissance individuelle en longueur des brins de laine chez 7 Brebis Ile-de-France avant thyroïdectomie (I à 2 mois) et après thyroïdectomie, d'abord sans traitement à la thyroxine ( 2 à 4 mois), puis avec traitement à la DLthyroxine par injections intraveineuses biquotidiennes (I mois).

Après thyroidectomie, cette vitesse de croissance ne représente plus, selon les animaux, que 55 à 77 pour cent de la valeur initiale et demeure constante tant qu'ils sont privés de thyroxine. Avec $50 \gamma / \mathrm{kg} / \mathrm{j}$ de DL-thyroxine le taux de croissance normal de la laine est presque rétabli, tandis qu'avec Ioo et $150 \gamma / \mathrm{kg} / \mathrm{j}$ il est dépassé, mais de peu : $19 \mathrm{p}$. 100 au maximum. On en déduit que la dose physiologique de DL-thyroxine est comprise entre 5o et roo $\gamma / \mathrm{kg} / \mathrm{j}$. Il semble, en outre, que plus la vitesse de croissance de la laine diminue à la suite de la thyroïdectomie, plus elle est difficile à rétablir par l'administration de thyroxine.

On n'a observé aucune modification significative du diamètre des brins de laine.

On sait que les hormones thyroïdiennes agissent sur la croissance de la laine : la thyroïdectomie est suivie d'une diminution de la quantité de laine produite par unité de surface de peau (30 à $40 \mathrm{p}$. IOo) tandis que l'administration de thyroxine rétablit cette production ou même l'accroît par rapport à la normale de 6 à $29 \mathrm{p}$. Ioo suivant les races ovines et les méthodes expérimentales utilisées (Simpson, I924; Marston et PIfRCE, I932; Ferguson, I957-58; Ross et LEWIS, I958; HarT, I954, I955, I957, I958; GODFREY, I958 ; GODFREY et TRIBE, I959).

Mais bien que 1'on estime en général que l'action des hormones thyroïdiennes porte essentiellement sur la croissance en longueur de la laine et fort peu sur le diamètre et la densité folliculaire, qui sont les deux autres paramètres de sa production pondérale par unité de surface de peau, il n'existe aucune donnée précise sur les variations de la vitesse de croissance en longueur du brin de laine en fonction de la thyroxinémie. 
Or, grâce à l'emploi des radioisotopes, on peut maintenant suivre avec une très grande précision la vitesse de croissance individuelle des poils (ROUGEOT, I959; Downes et LyNE, I959).

Aussi nous a-t-il semblé intéressant d'appliquer cette nouvelle méthode pour étudier les variations de la vitesse de croissance de la laine chez les Brebis au cours des trois phases expérimentales suivantes :

- avant thyroidectomie ;

- après thyroïdectomie sans supplémentation de 10L-thyroxine ;

- au cours des injections, quelques mois plus tard, de 50, IOO, I50 $\gamma / \mathrm{kg} / \mathrm{jour}$ de DL-thyroxine.

\section{MATÉRIEL, ET MÉTHODE}

$$
\text { I. - Les animaux. }
$$

Le choix s'est porté sur des Brebis de la race "Ile-de-France " dont la toison est parfaitement homogène puisqu'elle n'est composée que de laine à l'exclusion de tout autre type de poil (jarre, crin, etc.) ; la croissance de cette laine est continue et même constante lorsque les animaux adultes sont maintenus en conditions physiologiques stables (ROvGEOT, 1959).

Les 7 Brebis choisies étaient âgées de I à 2 ans, donc en fin de croissance, lorsque l'expérience a commencé. Elles furent soumises à un régime alimentaire de composition constante et gardées en local clos où on put les soustraire dans une certaine mesure, aux variations des facteurs saisonniers (lumière, température). Leur poids fut contrôlé toutes les semaines.

\section{2. - Thyrödectomie et injection consécutive de DL-thyroxine.}

Quand on eut vérifié pendant i à 2 mois que les Brebis s'adaptaient parfaitement à leur nouveau régime (pas de maladie, croissance corporelle régulière) on pratiqua sur elles la thyroïdectomie, en veillant à la suppression totale de l'isthme et en prenant soin de ligaturer les vaisseaux glandulaires le plus loin possible de la glande.

Le test in vivo à ${ }^{131}$ I effectué en fin d'expérience prouva que l'ablation de la glande avait été complète chez tous les animaux : aucune accumulation élective de radioiode ne fut constatée à l'emplacement de la thyroide.

Deux mois et demi à quatre mois après la thyrö̈dectomie, on administra aux Brebis de la DL. thyroxine de synthèse "Hoffman Laroche "par injections biquotidiennes dans la veine jugulaire. Trois Brebis reçurent $50 \mathrm{r} / \mathrm{kg} / \mathrm{jour}$ de DI.-thyroxine, 2 en reçurent $100 \mathrm{\gamma} / \mathrm{kg} / \mathrm{jour}$ et les 2 dernières I $50 \mathrm{\gamma} / \mathrm{kg} / \mathrm{jour}$.

\section{3. - Mesure de la variation de la vitesse de croissance individuelle des brins de laine.}

La croissance de la laine a été suivie en marquant chacun des brins appartenant à une même mèche de repères radioactifs, datés avec précision, obtenus en injectant par voie sous-cutanée, en un même point de la peau, de très faibles doses de radiocystine ${ }^{35} \mathrm{~S}$, soit 1o $\mu \mathrm{C}$ au maximum. En effet, la radiocystine injectée de cette façon participe immédiatement à la synthèse de la kératine de la laine poussant dans la zone d'injection et marque chacun des brins de points radioactifs correspondant, à quelques heures près, à la date de la piqûre (RougEoT, I959).

Dans le cas présent, les injections de radiocystine ont été faites tous les I4 jours en un point tatoué sur le dos de chacune des 7 Brebis. 15 jours après la dernière piqûre la laine du centre de la zone d'injection fut rasée. Ene dizaine de brins de laine furent prélevés dans chaque échantillon, puis étendus sur de grandes lames de verre et recouverts d'émulsion sensible coulable Ilford $G_{5}$; l'intervalle, entre 2 points radioactifs successifs révélés par l'autoradiographie, fut déterminé par projection microscopique, en mesurant, à l'aide d'un curvimètre, la distance entre les marges apicales de chaque point, à $2 \mathrm{~mm}$ près $(0, \mathrm{I} \mathrm{mm}$ près en grandeur réelle).

Comme les brins de laine appartenant à une même mèche, croissent en synchronisme avec des vitesses différentes les unes des autres, ils sont de longueurs inégales (RovgEOT, 1959. Downes et LYNE I96r) : aussi, dans les calculs de moyennes de croissance entre points radioactifs correspondant à 2 injections successives, avons-nous été amenés à exprimer les longueurs des intervalles en pourcentage de la distance comprise entre le premier et le dernier point radioactif. 


$$
\text { 4. - Mesure des diametres. }
$$

A la fin de chacune des 3 phases expérimentales nous avons prélevé par rasage une mèche de laine dans la zone d'injection et mesuré les diamètres de l'extrémité basale de zoo brins de chacune de ces mèches par projection microscopique au grandissement 500 (RovgEoT, I954).

\section{RÉSULTATS}

\section{I. - Variations du poids des brebis (figure $\mathbf{I}$ )}

La thyroïdectomie provoqua d'abord une augmentation sensible du gain de poids ( $I, I 70 \mathrm{~kg} /$ semaine au lieu de $0, I 50 \mathrm{~kg} / \mathrm{semaine})$ puis il y eut une légère tendance à la stabilisation 2 à 3 mois après la thyroïdectomie selon les individus.

Les injections de DL-thyroxine entraînèrent une chute rapide du poids des Brebis : les Brebis du lot à $50 \%$ subirent une chute de croissance pendant 3 semaines au taux moyen de $2,200 \mathrm{kgs} / \mathrm{semaine}$, celles du lot à Ioo $\gamma$, pendant 4 semaines au taux moyen de $2,800 \mathrm{kgs} / \mathrm{semaine}$ et celles du lot à $\mathrm{I}_{5} \mathrm{\gamma}$ pendant 5 semaines au taux moyen dè $3,300 \mathrm{kgs} / \mathrm{semaine}$. Il en résulta qu'en traitant les Brebis avec I50 $\gamma / \mathrm{kg} / \mathrm{jour}$ de DLthyroxine, leur poids tomba bien en dessous de celui qui existait avant la thyroïdectomie ( $5 \mathrm{kgs}$ pour la $\mathrm{n}^{0} 5,8 \mathrm{kgs}$ pour la $\mathrm{n}^{\circ} 8$ ) alors que le poids des Brebis recevant 50 ou roo $\gamma / \mathrm{kg} / \mathrm{j}$ rejoignit, à peu de chose près, le niveau qui aurait été atteint si elles n'avaient subi aucun traitement. Après cette chute de poids, le taux de croissance reprit sa valeur normale dans le lot à $50 \gamma$, le poids se stabilisa au niveau auquel il était descendu dans le lot à roo $\gamma$ et présenta des variations très irrégulières dans le lot à I5o $\gamma$.
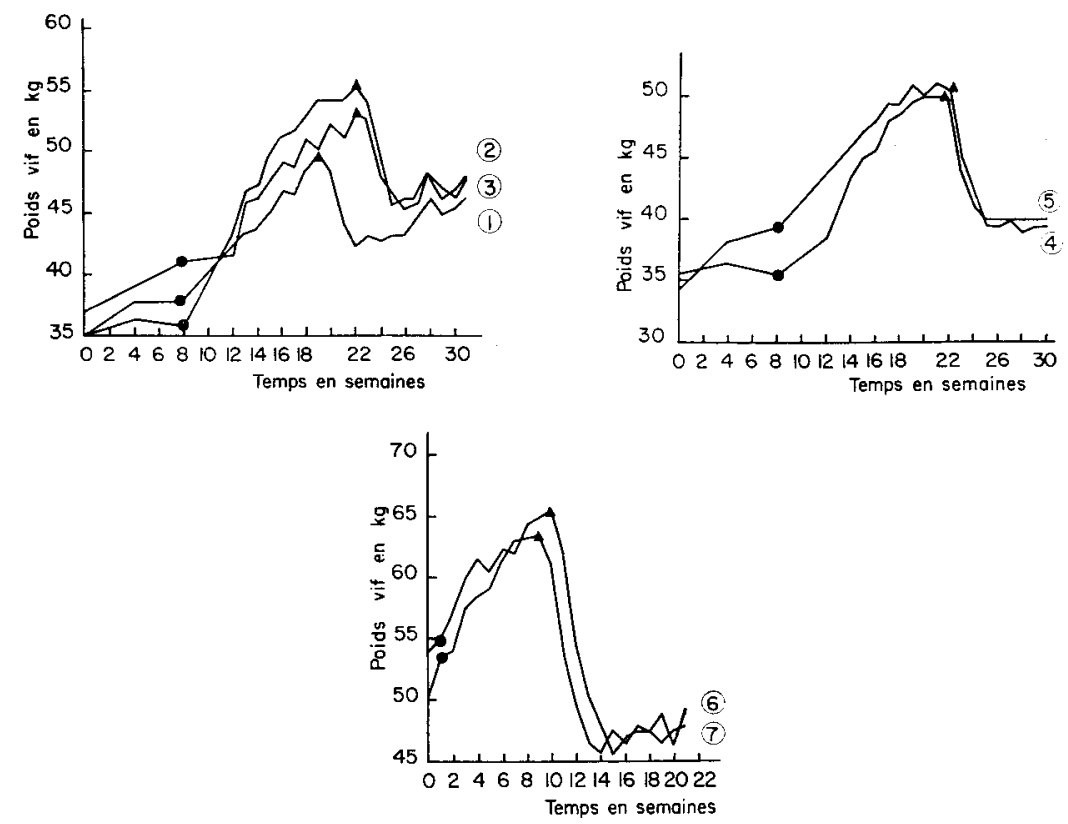

Fig. I. - Variation du poids des brebis.

- date de la thyroïdectomie

- date de la rre injection de DL-thyroxine. 


\section{2. - Taux de croissance de la laine (figure 2)}

Pendant la phase d'observation et de contrôle qui précéda la thyroïdectomie, la croissance de la laine étant constante (ROUGEOT, I959; DownEs et LyNE, I959), il a suffi d'obtenir 3 ou 4 points radioactifs pour déterminer le taux de croissance en longueur de la laine durant ce temps. Ce taux de croissance que l'on considère comme normal, par rapport à ceux qui vont suivre, a été indexé Ioo pour chacune des Brebis afin de pouvoir comparer commodément les animaux entre eux.

Après thyroïdectomie, le taux de croissance de la laine décroît progressivement pendant une quinzaine de jours car l'élimination des hormones thyroïdiennes ne se fait qu'au bout d'un certain temps. Après cette courte période transitoire, la croissance fut à nouveau constante, ce qui se traduisit sur les graphiques par les paliers situés à des niveaux bien différents selon les individus; les valeurs moyennes de ces paliers sont : 55 p. IOO ( $\mathrm{n}^{\circ} 3$ et 7$) ; 67$ p. IOO $\left(\mathrm{n}^{0} \mathrm{I}\right) ; 68$ p. IOO $\left(\mathrm{n}^{\circ} 6\right) ; 75$ p. IOO $\left(\mathrm{n}^{0} 2\right) ; 75$ p. IOO (no 5$)$ et 77 p. IOO $\left(\mathrm{n}^{0} 4\right)$.

E, in administrant $50 \mathrm{\gamma} / \mathrm{kg} / \mathrm{j}$ de DL-thyroxine le taux normal de croissance est presque atteint : 82 p. I00 $\left(\mathrm{n}^{0} 3\right) ; 96$ p. Ioo $\left(\mathrm{n}^{0} 2\right)$ et 99 p. Ioo $\left(\mathrm{n}^{0} \mathrm{I}\right)$. Avec $100 \mathrm{\gamma} / \mathrm{kg} / \mathrm{j}$ on dépasse de $I 7 \mathrm{p}$. Ioo $\left(\mathrm{n}^{\circ} 4\right)$ et de $\mathrm{I} 9 \mathrm{p}$. roo $\left(\mathrm{n}^{\circ} 5\right)$ le taux normal de croissance, l'augmentation étant du même ordre de grandeur avec $150 \mathrm{\gamma} / \mathrm{kg} / \mathrm{j}:$ I8 p. Ioo $\left(\mathrm{n}^{\circ} 6\right)$ et 5 p. IOO $\left(n^{\circ} 7\right)$.

\section{3. - Diamètres}

La thyroïdectomie, comme les injections consécutives de différentes doses de DL-thyroxine, n'ont entraîné aucune modification significative du diamètre le long du brin de laine.

TABIEAU I

Diamètre des brins de laine en fonction des différentes phases expérimontales et des doses de DL-thyroxine administrées aux brebis

\begin{tabular}{|c|c|c|c|c|}
\hline \multirow{3}{*}{$\begin{array}{c}\text { Doses } \\
\text { re DL-thyroxine }\end{array}$} & \multirow{3}{*}{$\begin{array}{l}\text { Numéros } \\
\text { des Brebis }\end{array}$} & \multicolumn{3}{|c|}{ Diamètres en microns } \\
\hline & & \multirow{2}{*}{ Avant thyroïdectomie } & \multicolumn{2}{|c|}{ Apres thyroïdectomie } \\
\hline & & & Sans thyroxine & Avec thyroxine \\
\hline $50 \mathrm{\gamma} / \mathrm{kg} / \mathrm{j} \ldots \ldots \ldots$ & $\begin{array}{l}1 \\
2 \\
2 \\
3\end{array}$ & $\begin{array}{l}25,6 \doteqdot 0,24 \\
25,0 \div 0,11 \\
25,2 \div 0,18\end{array}$ & $\begin{array}{l}27,2 \pm 0,23 \\
26,6 \pm 0,13 \\
25,4 \pm 0,17\end{array}$ & $\begin{array}{l}20,8=1 ; 0,21 \\
30,2= \pm 0,24 \\
20,2=0,10\end{array}$ \\
\hline $100 \gamma / \mathrm{kg} / \mathrm{j} \ldots \ldots$ & $\begin{array}{l}4 \\
5\end{array}$ & $\begin{array}{l}25, \pm \pm 0,18 \\
21,0 \pm 0,14\end{array}$ & $\begin{array}{l}22,6 \pm 0,12 \\
19,4 \pm 0,11\end{array}$ & $\begin{array}{l}24,2 \pm 0,20 \\
23,6 \pm 0,2\end{array}$ \\
\hline $150 \mathrm{r} / \mathrm{kgg} / \mathrm{j} \ldots \ldots$ & $\begin{array}{l}6 \\
7\end{array}$ & $\begin{array}{l}26,2 \div 0,15 \\
23,2=0,17\end{array}$ & 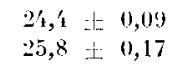 & $\begin{array}{l}20,2 \pm \pm 0,09 \\
25, \underline{2} \pm 0,06\end{array}$ \\
\hline
\end{tabular}



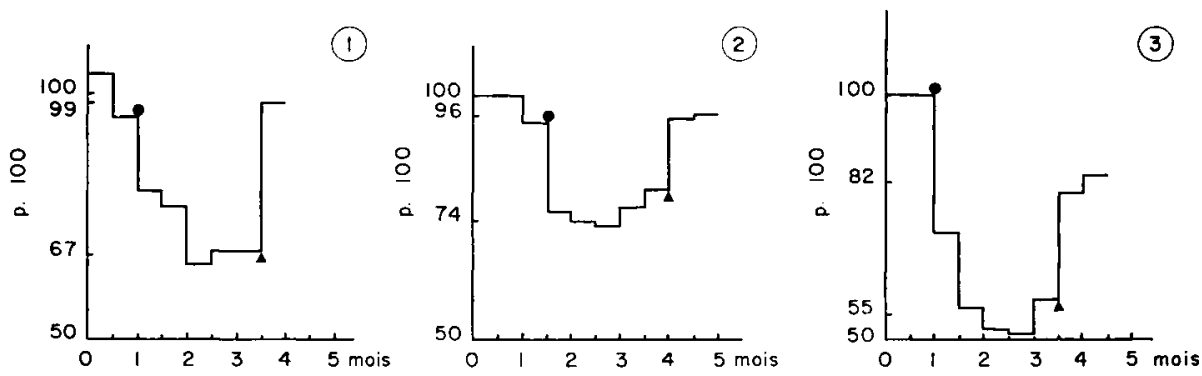

DL - THYROXINE , $50 \mathrm{r} / \mathrm{kg} / \mathrm{i}$
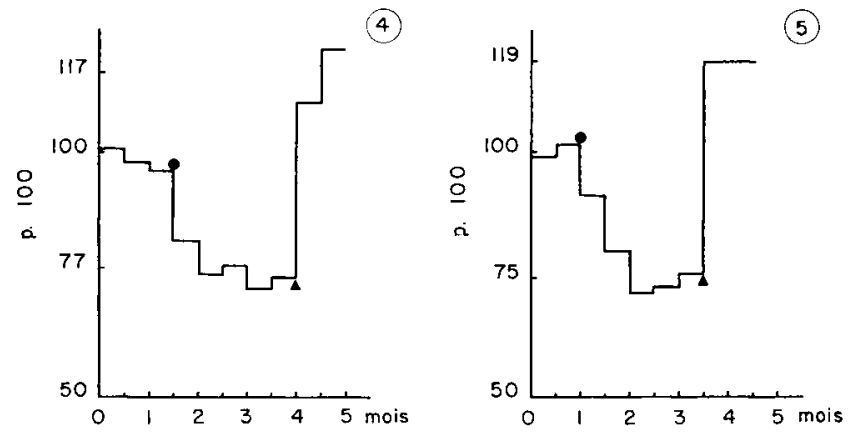

DL - THYROXINE, $100 \gamma / \mathrm{Kg} / \mathrm{j}$
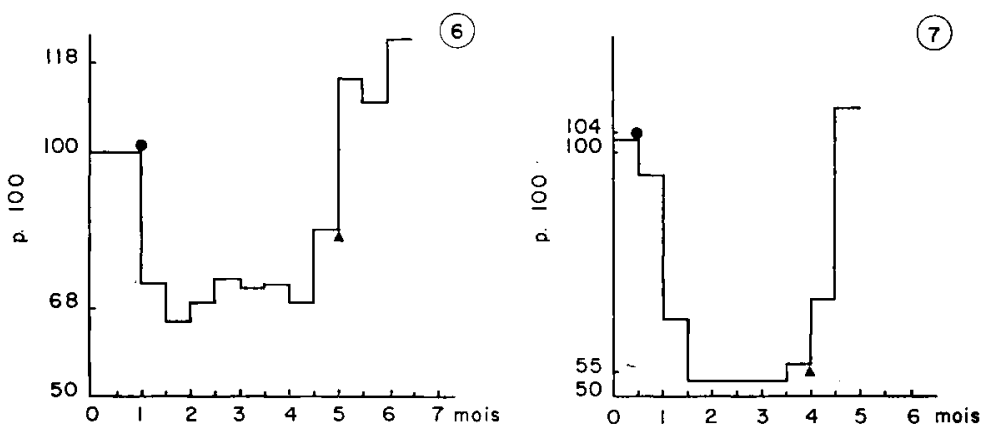

$\mathrm{OL}$ - THYROXINE, $150 \gamma / \mathrm{Kg} / \mathrm{i}$

Fig. 2. - Variation du tanx de croissance de la laine.

- date de la thyroïdertomie

a date de la $\mathbf{I}^{\mathbf{0}}$ injection de DL-thyroxine

Les chiffres des ordonnées indiquent les valeurs moyennes des paliers correspondant aux 3 phases ixpérimentales. 


\section{DISCUSSION}

La dose de $5^{\circ} \gamma / \mathrm{kg} / \mathrm{j}$ de DL-thyroxine, qui rétablit le taux de croissance normal du poids corporel après la phase d'amaigrissement, peut être considérée comme dose physiologique pour les Brebis Ile-de-France. Par contre, les doses de Ioo et $150 \mathrm{\gamma} / \mathrm{kg}$ j de DL-thyroxine entraînent un état d'hyperthyroïdisme : c'est très net avec la dose de $I 50 \mathrm{\gamma} / \mathrm{kg} / \mathrm{j}$ qui, après avoir produit un amaigrissement beaucoup plus prononcé et pendant plus longtemps que les autres doses, provoque des variations de poids incohérentes chez les Brebis : mais déjà avec roo $\gamma / \mathrm{kg} / \mathrm{j}$ de DL-thyroxine on ne parvient pas à rétablir le taux de croissance normal après la phase d'amaigrissement.

On retrouve une action comparable de ces trois doses différentes de DL-thyroxine sur le taux de croissance en longueur de la laine. La dose de $50 \% / \mathrm{kg} / \mathrm{j}$ rétablit presque le taux de croissance initial de la laine, tandis qu'avec celles de Ioo et I50 $\gamma / \mathrm{kg} / \mathrm{j}$ ce taux est nettement dépassé. Mais avec $\mathbf{r}_{50} \gamma / \mathrm{kg} / \mathrm{j}$ de I)L-thyroxine, la laine ne croît pas plus rapidement qu'avec Ioo $\gamma / \mathrm{kg} / \mathrm{j}$. On peut donc estimer que la dose physiologique de DL-thyroxine est comprise entre 50 et Ioo $\gamma / \mathrm{kg} / \mathrm{j}$ et affirmer qu'avec $\mathrm{I}_{50} \mathrm{\gamma} / \mathrm{kg} / \mathrm{j}$ les animaux sont déjà en état d'hyperthyroïdisme. On ne peut toutefois préjuger de $1^{\prime}$ action de doses très supérieures à $15^{\circ} \gamma / \mathrm{kg} / \mathrm{j}$ sur la vitesse de croissance de la laine.

La thyroïdectomie réduit le taux de croissance de la laine dans des proportions très différentes selon les Brebis, et les mêmes doses d'hormone n'ont pas le même effet dans le rétablissement ou le dépassement de ce taux : en particulier si 1'on considère les 2 Brebis où le taux de croissance subit la diminution la plus forte après la thyroïdectomie, soit $55 \mathrm{p}$. IOO, on constate que le taux de croissance normal n'est pas tout à fait rétabli chez la Brebis $n^{\circ} 3$ qui reçoit $50 \gamma / \mathrm{kg} / \mathrm{j}$ de DL-thyroxine et n'est pas dépassé de beaucoup chez la Brebis no 7 qui reçoit $5_{50} \gamma / \mathrm{kg} / \mathrm{j}$. Tout semble donc se passer comme si la reprise ou l'augmentation de la vitesse de croissance de la laine, à la suite de l'administration de thyroxine, était d'autant plus difficile à réaliser que la thyrö̈dectomie avait été suivie d'une réduction plus forte de cette vitesse de croissance. On ne peut pourtant affirmer que cela soit dû uniquement à l'activité thyroïdienne propre à chaque animal car on peut concevoir que les follicules laineux répondent différemment à une même dose d'hormone.

Il est vrai aussi que l'amplitude de cette réponse des follicules pourrait être réglée par le niveau d'activité thyroïdienne initial de l'animal, avec lequel ils fonctionnaient normalement.

Si les hormones thyroïdiennes agissent nettement sur la vitesse de croissance en longueur de la laine, bien que modérément, surtout dans le cas de 1'hyperthyroïdisme, leur suppression ou leur excès ne provoque aucun changement mesurable du diamètre ; ceci mérite d'être souligné car il est notoirement connu que les variations du régime alimentaire agissent à la fois, et dans le même sens, sur le taux de croissance en longueur et sur le diamètre de la laine : il en est de même dans le cas de la sympathectomie (FERGUSON, I949) qui entraîne une augmentation de la croissance en longueur et en diamètre par vasodilatation des capillaires de la peau. On peut donc supposer que les hormones thyroïdiennes, suivant leur concentration dans le sang, introduisent des variations dans le nombre des mitoses dans le bulbe folliculaire, 
peut-être dans la taille des cellules produites, certainement en tout cas, dans la quantité de kératine synthétisée au niveau du col du follicule ; mais le diamètre du follicule, et en particulier celui de la gaine interne qui fait fonction de filière continue, ne subirait aucune modification. Ceci implique des modifications morphologiques et structurales de la laine, d'autant plus intéressantes à étudier, qu'elles ne dépendent que d'un seul des detux paramètres entrant en jeu dans sa croissance.

$$
\text { Reçu en dicembre I96ז. }
$$

\section{SUMMARY}

THE EFFECT OH THYROID HORMONES ON THE LENGITT GROWTII OF THE WOOL FIBER.

With the use of radiocystine the rate of growth in length of individual wool fibres was studied with precision in 7 Ile-de-France ewes before thyroidectomy ( $\mathrm{r}$ month), and after thyroidectomy, first without thyroxine (I-2 months), then with thyroxine injected intravenously twice a day (I-2 months).

After thyroildectomy, the rate of wool growth falls, according to the animals, to 55 to 77 per cent of the initial level; then it remains constant as long as thyroxine is not given : with $50 \mathrm{\gamma} / \mathrm{kg} / \mathrm{day}$ of DL-thyroxine the normal rate of wool growth is nearly restored, whereas with roo and $150 \gamma / \mathrm{kg} / \mathrm{day}$ it is passed, but only slightly, by ig per cent maximum. So, it is thought that the physiological dose of DI.-thyroxine lies between 50 and $100 \mathrm{\gamma} / \mathrm{kg} /$ day. Moreover, it seems that the greater the decrease of the rate of wool growth caused by the thyroidectomy, the greater the difficulty of restoring it by thyroxine.

No significative change occured in diancter of wool fibres.

\section{RÉFÉRENC'ES BIBLIOGRAPHIQUES}

Downes A. M., I.YNE A. G., I959. Measurement of the rate of growth of wool using cystine labelled with sulphur 35. Nature, 12, I885-1886.

Downes A. M., Lyne A. G., I96r. Studies on the rate of wool growth using ${ }^{35}$ S cystine. Aust. J. Biol.

Sci., 14, I $20-130$.

Ferguson K. A., 1949. The effect of sympathectomy on wool growth. Aust. J. Sici. Res., 2, $43^{8-443 .}$

Ferguson K. A., 1957-58. The infuence of thyroxine on wool growth. Proc. N. Z. Soc. Anim. Prod. 17-

18, I28.

Godfrey N. W., 1958. Thyroxine implantation and wool growth of sheep. Proc. Aust. Soc. Anim. Prod., 2, I $29^{-1} 30$.

Godfrey N. W., 'Tribe D. L., 1959. The effect of thyroxine implantation on wool growth. J. Agric. Sci.' 53, 369-375.

HART D. S., 1954. Augmentation of wool growth by L-thyroxine and triodo-thyronine in sheep. Nature 174, IOI8.

Hart D. S., I955. Photoperiodic and hormone response of wool growth in sheep. Proc. N. Z. Soc. Anim. Prod., 15, 57 .

HarT D. S., I957. Stimulation of wool growth by thyroxine implantation. N. Z. J. Sci. Tech., 38 A, 87 I.

HaR'T D. S., I958. Response of sheep to thyroxine implantation. Proc. N. Z. Soc. Anim. Prod., 18, i53-I60.

Marston H. R., Pierce A. W., I932. The effect following thyroidectomy in sheep. Aust. J. Exp. Biol. Med. Sci., 10, 203 .

Rougeot J., I954. Les mesures du diamètre des brins de laine. Bull. Inst. Texi. Fr., 44, 42-51.

Rougeot J., 1959. Mesure de la croissance individuelle des brins de laine à l'aide de radiocystine. Ann. Zootech., 2, I 75-177.

Ross D. A., Lewis K. I., 1958. The effect of thyroxine on the Romney two-tooth. Proc. N. Z. Soc. Anim. Prod., 17-18, I4I.

Simpson S., r924. Effect of thyroïdectomy on cutaneous system in sheep and goats. Quart. J. Exp.physiol. $14,185$. 